\title{
Enterocytozoon bieneusi detected by molecular methods in raw sewage and treated effluent from a combined system in Brazil
}

\author{
Sandra Yamashiro', Vagner Ricardo da Silva Fiuza², \\ Ângela Therezinha Lauand Sampaio Teixeira ${ }^{3}$, Nilson Branco', Carlos Emílio Levy3, \\ Isabel Cristina Vidal Siqueira de Castro', Regina Maura Bueno Franco ${ }^{1 /+}$
}

\author{
${ }^{1}$ Universidade Estadual de Campinas, Instituto de Biologia, Departamento de Biologia Animal, \\ Laboratório de Protozoologia, Campinas, SP, Brasil \\ ${ }^{2}$ Universidade Estadual de Campinas, Faculdade de Engenharia Civil, Arquitetura e Urbanismo, \\ Departamento de Saneamento e Ambiente, Campinas, SP, Brasil \\ ${ }^{3}$ Universidade Estadual de Campinas, Faculdade de Ciências Médicas, Departamento de Patologia Clínica, \\ Hospital de Clínicas, Campinas, SP, Brasil
}

BACKGROUND Enterocytozoon bieneusi are the most common microsporidia associated with different clinical manifestations such as diarrhoea, respiratory tract inflammation and acalculous cholecystitis, especially in immunocompromised patients. Infection usually occurs by ingestion of food and water contaminated with spores, but can also result from direct contact with spores through broken skin, eye lesions, and sexual transmission, depending on the microsporidian species. Although there are reports of $E$. bieneusi found in humans and animals in Brazil, there are no published studies of environmental samples examined by molecular methods.

OBJECTIVES The purpose of this study was to verify the presence of E. bieneusi in raw sewage and treated effluent from a combined system by molecular methods.

METHODS Raw sewage and treated effluent samples collected from a combined system were analysed for the presence of $E$. bieneusi using the internal transcriber spacer (ITS) region of E. bieneusi by nested polymerase chain reaction.

FINDINGS The analysis revealed E. bieneusi presence and a novel genotype (EbRB) in one raw sewage sample and one treated effluent.

MAIN CONCLUSIONS The presence of E. bieneusi in final effluent indicates that the combined system may not remove microsporidian spores. This study is the first report of E. bieneusi in environmental samples in Brazil.

Key words: sewage - Enterocytozoon bieneusi - PCR - treated effluent - combined system - Brazil

Microsporidia represent a group of obligate intracellular parasites nested within the kingdom Fungi, with 1300 to 1500 species in 187 genera (Vávra \& Lukes 2013). These ubiquitous organisms have been found in a wide range of vertebrate and invertebrate hosts with at least 17 species reported in humans, in which they can cause the opportunistic disease microsporidiosis (Fayer \& Santín-Duran 2014). Faecal-oral transmission by ingestion of food and water contaminated with spores is the most likely route of transmission to humans (Didier $\&$ Weiss 2011), whose infections are of commercial, ecological, and medical significance (Weiss \& Becnel 2014). Spores are resistant to the environment and may exhibit long survival times (Li et al. 2003).

Microsporidiosis has been reported in all continents except Antarctica (Weiss 2014), primarily in severely immunocompromised individuals (e.g., HIV/AIDS and transplant patients) (Galván et al. 2011). Diarrhoeal episodes in patients with AIDS can range from $30 \%$ to $70 \%$ (Field \&

\footnotetext{
doi: 10.1590/0074-02760160435

+ Corresponding author:mfranco@unicamp.br

Received 28 September 2016

Accepted 4 March 2017
}

Milner Jr 2015). However, cases in HIV-negative people, tourists, and the elderly are increasing (Galván et al. 2011).

Enterocytozoon bieneusi are the most common microsporidia associated with different clinical manifestation such as diarrhoea, respiratory tracts inflammation and acalculous cholecystitis (Didier \& Weiss 2006). Spores have been detected in various environmental samples, including surface water (Sparfel et al. 1997, Dowd et al. 1998), swimming pools (Fournier et al. 2002), wastewater samples (Ayed et al. 2012, Ma et al. 2016), and crop irrigation water (Cotte et al. 1999). Moreover, a foodborne outbreak of gastrointestinal illness was caused by food ingestion in over 100 people was associated with the consumption of E. bieneusi-contaminated vegetables in Sweden (Decraene et al. 2012).

The epidemiology of microsporidiosis in humans in developing countries is poorly understood (HenriquesGil et al. 2010, Li et al. 2012); hence there is a great need for clinical and environmental research to better understand E. bieneusi distribution and its risks to human health. In Brazil, nine studies have used molecular methods to detect this pathogen in HIV-infected patients presenting chronic diarrhoea, and animals including cattle, pigs, chickens, coatis, pigeons, and exotic birds, which highlights the potential risk of transmission to healthy humans (Brasil et al. 1998, 2000, Feng et al. 2011, Lallo 
et al. 2012a, b, Fiuza et al. 2015, 2016a, b, Cunha et al. 2016). However, no information regarding E. bieneusi presence in environmental samples in Brazil is available.

During January 2013 to June 2014, Costa et al. (2013) verified several cases of microsporidiosis among transplant patients and HIV+ individuals attended by the University Clinical Hospital (University of Campinas, Campinas, Brazil) by microscopy techniques.

In this period, raw sewage and treated effluent samples from a combined system that receives and treats the hospital effluent were preserved to verify the presence of $E$. bieneusi, since diarrhoea was the most prominent clinical manifestation in these patients. Furthermore, E. bieneusi is the microsporidian species most commonly found in humans. Molecular analysis was performed and revealed the presence of $E$. bieneusi DNA. In addition, a novel $E$. bieneusi genotype (EbRB) was found in one raw sewage sample and one treated effluent sample. Our findings are of great importance to public health, as this is the first report of molecular methods used to determine the presence of $E$. bieneusi in environmental samples in Brazil.

\section{MATERIALS AND METHODS}

Wastewater samples - Wastewater sampling was performed from January, 2013, to June, 2014, every two weeks, regarding the hydraulic retention time (HRT) of each reactor. Samples from raw sewage $(n=18)$ and treated effluent $(\mathrm{n}=18)$ were collected in plastic bottles. The detection of E. bieneusi was conducted at the Laboratory of Protozoology, Institute of Biology, University of Campinas.

The raw sewage samples were subjected to a centrifugeconcentration technique described by Cantusio et al. (2006) with minor modifications, including centrifugation at 1500 $\mathrm{x} g$ for $15 \mathrm{~min}$, and using 1\% Tween 80 for homogenisation of samples. Treated effluent samples were subjected to the membrane filtration technique of Franco et al. (2001), using $1 \%$ Tween 80 to elute membrane surfaces and a double centrifugation step at $1500 \mathrm{x} g$ for $15 \mathrm{~min}$.

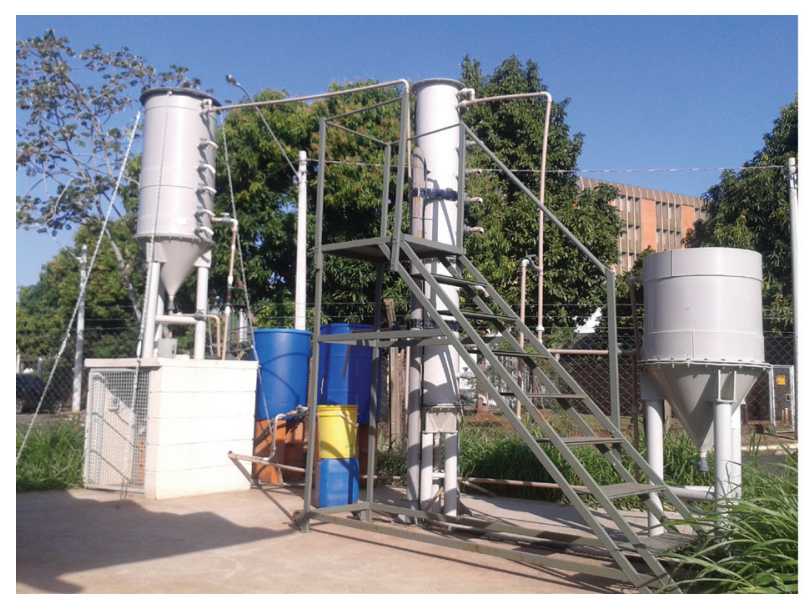

Fig. 1: pilot combined system of wastewater treatment: sewage reservoir (blue tank); from left to right: anaerobic/anoxic filter (AAF) followed by aerated submerged biofilter (ASB) and decanter (DEC) located at the Biological Process Laboratory in University of Campinas (Campinas, São Paulo, Brazil).

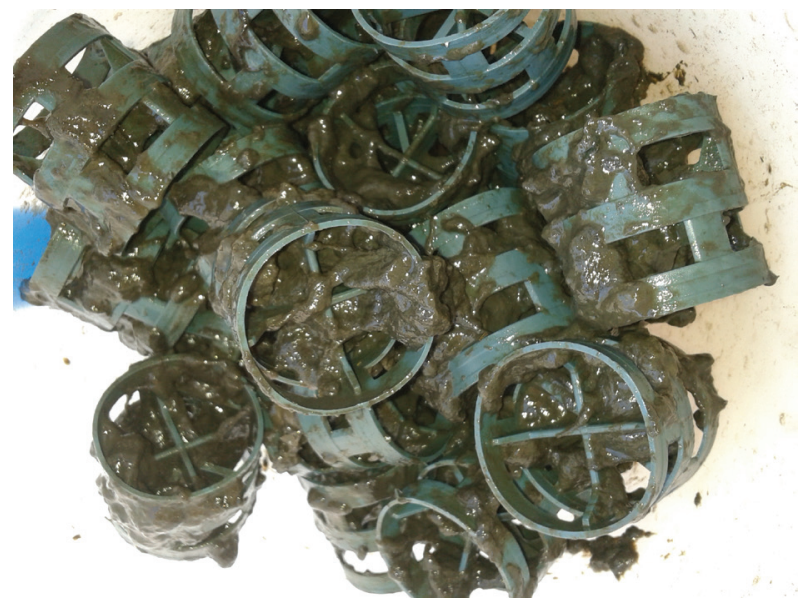

Fig. 2: "Pall rings" in the aerated submerged biofilter.

Region studied - The combined system was operated at a pilot scale in the Biological Processes Laboratory (LABPBIO), located at the University of Campinas, Campinas, Southeast Brazil.

This system was filled with raw sewage that received a discharge of effluent that drains a region of the University of Campinas campus comprising a metropolitan reference hospital, a nursery, financial banks, several cafeterias, and three university restaurants. Over ten thousand people circulate through this area daily.

Combined system - The combined system consisted of one trickling filter composed of the following units: an anaerobic/anoxic filter (AAF) with a volume of 298.7 L, an aerated submerged biofilter (ASB) with a volume of $131.5 \mathrm{~L}$, and a decanter (DEC) with a volume of 188.5 L (Fig. 1). Both reactors (AAF and ASB) were fulfilled with "pall ring" type rings (Fig. 2) produced in recycled polypropylene with a dimension of $38 \mathrm{~mm} \times 38 \mathrm{~mm}$ (diameter $x$ height), and a surface area of $128 \mathrm{~m}^{2} \cdot \mathrm{m}^{-3}$, and a "free space index" of $89 \%$.

A volume of $700 \mathrm{~L}$ of raw sewage is treated daily by the combined system with a flow rate of $25 \mathrm{~L} / \mathrm{h}$ (Fig. 3).

DNA extraction - DNA extraction of microsporidian spores was performed using the ZR Fungal/Bacterial $\mathrm{DNA}^{\circledR}$ kit (Zymo Research, Irvine, CA, USA) according to the manufacturer's instructions. After extraction, 100 $\mu \mathrm{L}$ DNA was stored at $-20^{\circ} \mathrm{C}$.

Nested polymerase chain reaction (PCR) and purification - A nested PCR protocol (Buckholt et al. 2002) was used to amplify two fragments (435 and $390 \mathrm{bp}$ ) of the internal transcriber spacer (ITS) region of the $E$. $b i$ eneusi rRNA gene, with the following primer sets: EBITS3: (5'- GGT CAT AGG GAT GAA GAG -3') and EBITS4: (5'- TTC GAG TTC TTT CGC GCT C -3'), for the first PCR; EB-ITS1: (5'- GCT CTG AAT ATC TAT GGC T -3') and EB-ITS2.4: (5'- ATC GCC GAC GGA TCC AAG TG -3') for the second PCR.

The PCR reaction mixture contained Ultrapure ${ }^{\mathrm{TM}}$ DNase-free Distilled Water (Invitrogen, Carlsbad, CA, USA), 1 x PCR buffer (Sigma-Aldrich, St Louis, MO, USA), $1.5 \mu \mathrm{M} \mathrm{MgCl}_{2}$ (Sigma-Aldrich), $0.2 \mu \mathrm{M}$ of each 
Sewage reservoir $\rightarrow$ Anaerobic/anoxic filter $\rightarrow$ Aerated submerged biofilter $\rightarrow$ Decanter

Fig. 3: flow direction of the sewage in the combined system for wastewater treatment.

dNTP (Sigma-Aldrich), 2.5 U Taq DNA Polymerase (Sigma-Aldrich), $2.5 \mu \mathrm{L}$ of BSA $(0.1 \mathrm{~g} / 10 \mathrm{~mL}), 1 \mathrm{pmol}$ of each forward and reverse primer, and $5 \mu \mathrm{L}$ of extracted DNA in a total reaction volume of $50 \mu \mathrm{L}$. After denaturation at $94^{\circ} \mathrm{C}$ for $3 \mathrm{~min}$, the first PCR consisted of 35 cycles (denaturation at $94^{\circ} \mathrm{C}$ for $30 \mathrm{~s}$, annealing at $57^{\circ} \mathrm{C}$ for $30 \mathrm{~s}$, and extension at $72^{\circ} \mathrm{C}$ for $40 \mathrm{~s}$ ), followed by a final extension at $72^{\circ} \mathrm{C}$ for $10 \mathrm{~min}$. Conditions for the second PCR were identical to the first PCR, except for the following modifications: 30 cycles with an annealing temperature of $55^{\circ} \mathrm{C}$. Also, $5 \mu \mathrm{L}$ of amplified DNA produced from the first PCR was used as template.

Negative and positive controls were included in all PCR reactions. Positive control samples were obtained from faecal samples from immunocompromised patients positive for E. bieneusi from the Clinical Hospital. PCR products were subjected to electrophoresis in agarose gel $(2 \%)$ with the Low DNA Mass Ladder (Invitrogen), and visualised under UV light (UVDI-365®, Major Science) after staining the gel with GelRed $^{\circledR}$ (Biotium, Fremont, CA, USA).

All positive PCR products were purified using the ExoSAP-IT (USB Corporation, Cleveland, OH, USA), following the manufacturer's instructions. Both strands were sequenced using the inner primers utilising the Applied Biosystems ${ }^{\circledR} 3500$ Genetic Analyzer (Helixxa, São Paulo, Brazil).
Sequences obtained were aligned with reference sequences downloaded from GenBank using the program MEGA version 7 (Kumar et al. 2016) to determine $E$. bieneusi genotypes. Phylogenetic and molecular evolutionary analyses were made using MEGA version 7 (Kumar et al. 2016). Phylogenetic inference was determined by the neighbor-joining (NJ) method of Saitou and Nei (1987). Genetic distance was calculated with the Kimura 2-parameter model. The GenBank accession numbers of the reference sequences are: AF 101200, AY371286, AF348475, AY371283, AF242478, AY371281, AF076042, AY237223, AF101199, AF076040, AF076043, AY331007, EU153584, FJ439683, KY363360, JN997481, JN595887, JN997479, JN997480, DQ683749, JQ437575.

\section{RESULTS}

From the 18 raw sewage samples analysed by nested PCR (Table), three (16.6\%) were positive for $E$. bieneusi (AM1, AM3, AM27), whereas out of 18 treated effluent samples (Table), two (11.1\%) were positive (AM28, AM47) (Fig. 4).

After sequencing all five positive samples, the $E$. bieneusi sequence was verified in four samples (AM3, AM27, AM28, and AM47). Sequencing of samples AM27 and AM47 revealed a novel E. bieneusi genotype (EbRB). The closest match for EbRB is genotype S7 (FJ439683) (syn. CHY1 KT267289) (Fig. 5), isolated from humans in the Netherlands (ten Hove et al. 2009), with five nucleotide differences. These sequences were deposited in GenBank under Accession Numbers KY363360 and KY593707. AM1, AM3, and AM28 were not successfully sequenced; hence, it was not possible to determine their genotypes.

TABLE

Nested polymerase chain reaction (PCR) results of Enterocytozoon bieneusi and genotype in raw sewage and treated effluent samples from combined system for wastewater treatment

\begin{tabular}{|c|c|c|c|c|c|}
\hline Raw sewage samples & Amplification & Genotype & Treated effluent samples & Amplification & Genotype \\
\hline AM1 & + & Not determined & AM2 & - & - \\
\hline AM3 & + & Not determined & AM4 & - & - \\
\hline AM5 & - & - & AM6 & - & - \\
\hline AM7 & - & - & AM8 & - & - \\
\hline AM9 & - & - & AM10 & - & - \\
\hline AM13 & - & - & AM11 & - & - \\
\hline AM14 & - & - & AM12 & - & - \\
\hline AM15 & - & - & AM17 & - & - \\
\hline AM20 & - & - & AM18 & - & - \\
\hline AM21 & - & - & AM19 & - & - \\
\hline AM27 & + & EbRB & AM28 & + & Not determined \\
\hline AM33 & - & - & AM22 & - & - \\
\hline AM34 & - & - & AM23 & - & - \\
\hline AM35 & - & - & AM24 & - & - \\
\hline AM36 & - & - & AM26 & - & - \\
\hline AM37 & - & - & AM32 & - & - \\
\hline AM38 & - & - & AM47 & + & $\mathrm{EbRB}$ \\
\hline AM46 & - & - & AM48 & - & - \\
\hline
\end{tabular}

+: positive; -: negative; samples in bold: AM28 is correspondent to AM27. 


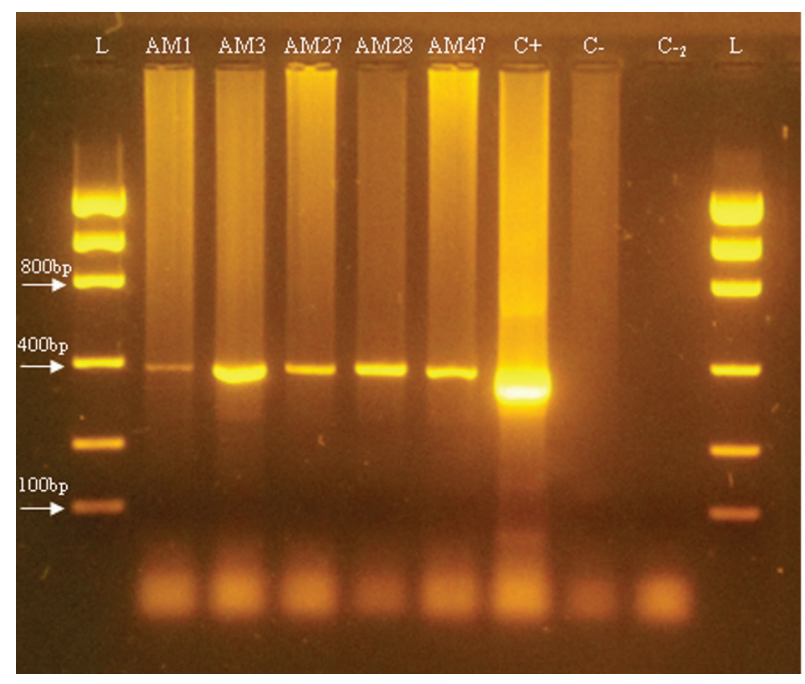

Fig. 4: agarose gel electrophoresis (2\%) showing amplification products from nested polymerase chain reaction (PCR) of Enterocytozoon bieneusi. L: Low DNA Mass Ladder; arrows indicate fragments of $100 \mathrm{bp}, 400 \mathrm{bp}$ and $800 \mathrm{bp}$; AM1; AM3 and AM27: raw sewage; AM28 and AM 47: treated effluent; $\mathrm{C}+$ : positive control; C-: first negative control; C-2: second negative control. Size of the fragment amplified from $E$. bieneusi $18 \mathrm{~S}$ rRNA: $390 \mathrm{bp}$.

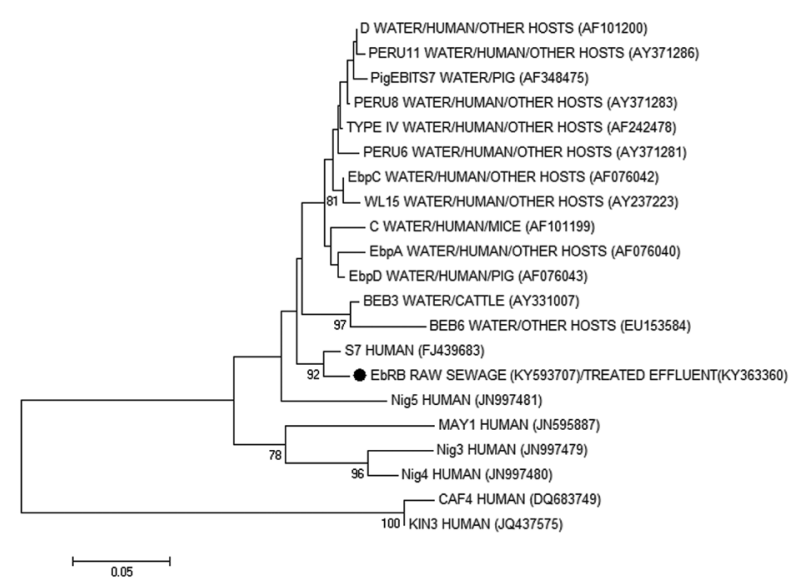

Fig. 5: phylogenetic relationships among the novel Enterocytozoon bieneusi genotype (EbRB, identified with a $\bullet$ ) found in raw sewage and treated effluent, and E. bieneusi genotypes previously identified in water, humans and/or other hosts as inferred by a neighbor-joining analysis of the internal transcriber spacer (ITS) rRNA gene sequence, based on genetic distances calculated by the Kimura two-parameter model. Bootstrap values of less than $75 \%$ are not shown.

\section{DISCUSSION}

In Brazil, prevalence surveys of HIV+ patients with diarrhoea showed that $5 \%, 27.5 \%$, and $46.1 \%$ were infected by microsporidia (Wuhib et al. 1994, Brasil et al. 1996, 2000). Microsporidian spores were also identified in $1.3 \%$ of immunosuppressed patients in the city of Goiânia (Goiás, Brazil) (de Souza Jr \& García-Zapata
2006), and in $36 \%$ of rheumatic disease patients undergoing anti-tumour necrosis factor therapy/anti-rheumatic drug treatment as compared to $4 \%$ of controls without diarrhoea (Aikawa et al. 2011). In 2013, Costa et al. (2013) described high positivity (40.3\%) for microsporidia in patients who underwent transplantation surgery in the University Clinical Hospital, including bone marrow (75\%), kidney (22\%), and liver (3\%) transplantation. These studies have shown that microsporidiosis is associated with chronic diarrhoea in immunocompromised patients and other immunosuppressed individuals in Brazil.

E. bieneusi was confirmed by PCR and electron microscopy as the only enteric pathogen found in five of 11 patients included in a cohort study of $40 \mathrm{HIV}+$ individuals with chronic diarrhoea in Rio de Janeiro state (Brasil et al. 2000). One $\mathrm{HIV}+$ patient exhibited chronic diarrhoea and a CD4 level $<100$ cells $/ \mathrm{mm}^{3}$ (Brasil et al. 1998).

Although molecular studies of microsporidia in Brazil are still incipient, some investigations have demonstrated that E. bieneusi is a common species parasitising animals in this country. There are reports in cattle (Fiuza et al. 2016a), pigs (Fiuza et al. 2015), sheep (Fiuza et al. 2016b), coatis (Lallo et al. 2012a), and birds (Lallo et al. 2012b, Cunha et al. 2016). These findings highlight the risk of human microsporidiosis by zoonotic transmission, since genotypes previously reported in both humans and animals were found in all animals surveyed in these studies, with the exception of coatis.

Many epidemiological studies have identified the consumption of untreated water as a potential risk factor for microsporidiosis in humans (Didier et al. 2004, Wumba et al. 2012), and E. bieneusi spores have been found in surface water at relatively high frequencies (Graczyk et al. 2010, Galván et al. 2013, Guo et al. 2014). Guo et al. (2014) found that $58.2 \%$ of storm water samples in the United States were positive for E. bieneusi using filtration and PCR. In China, Hu et al. (2014) found E. bieneusi in $31.5 \%$ of drinking water samples, after episodes of water contamination with carcasses of domestic pigs. Galván et al. (2013) reported that $61 \%$ of domestic effluent samples in Madrid, Spain, contained microsporidia such as Encephalitozoon intestinalis, Encephalitozoon cuniculi, Anncaliia algerae, and E. bieneusi (genotypes $\mathrm{C}$ and $\mathrm{D}$ ).

Although preliminary, our findings are relevant since this is the first report of E. bieneusi occurrence in environmental samples in Brazil. DNA of microsporidian spores was amplified from two samples (AM27 and AM28), revealing the presence of E. bieneusi in both raw sewage and corresponding treated effluent samples. This finding demonstrates that the combined system did not achieve complete removal of E. bieneusi spores. This issue can be attributed to the low sedimentation rate of the spores in the decanted liquid of the combined system, thus they remained in the treated effluent.

Until date, over 201 genotypes of E. bieneusi have been reported based on nucleotide polymorphisms in the ITS region of the rRNA gene (Fayer \& Santín-Duran 2014). While some genotypes are host-specific for humans, others show host specificity for animals, and some are zoonotic (Santín \& Fayer 2011). 
A novel E. bieneusi genotype (EbRB) was identified in a raw sewage (AM27) and a treated effluent (AM47) sample. The risks of human infection for this genotype are unknown. The closest match for this new strain is genotype $\mathrm{S} 7$, which was previously reported in a diabetic patient exhibiting severe diarrhoea and rectal bleeding (ten Hove et al. 2009), in horses (Santín et al. 2010), and in a yak (Bos grunniens) in a zoo in China (Li et al. 2015). Henriques-Gil et al. (2010) suggested that some genotypes of E. bieneusi circulate within a specific host species and are only occasionally transmitted to another host.

Furthermore, contaminated water is a possible source of infection for transplant and HIV+ patients. Sewage discharges are a significant risk factor for the introduction of human enteropathogens into surface waters (Graczyk \& Lucy 2007), and microsporidia spores are environmentally robust and ubiquitous in aquatic habitats (Graczyk et al. 2007). In addition, the present finding of $E$. bieneusi in treated effluent from the University Clinical Hospital is important from both public health and environmental standpoints because this final effluent flows into the Ribeirão das Pedras stream, which runs through several neighbourhoods, and is discharged into the Anhumas stream, a tributary of the Atibaia River, the main water supply of the city of Campinas.

By using E. intestinalis in their assays, Gerba et al. (2003) evaluated spore removal through a conventional water treatment process on a pilot scale, and reported a high removal efficiency (2.47 log) using coagulation and sedimentation steps followed by a mixed media filtration. However, spore size can influence the removal efficiency attained by water and wastewater treatment plants, and $E$. bieneusi spores are smaller than those of $E$. intestinalis.

Negative removal efficiencies were obtained for $E$. $b i$ eneusi in two plants (-100\%) by Cheng et al. (2011), who also reported high concentrations of microsporidian spores in inflowing wastewaters and in wastewater processing end-products (biosolids and final effluents) in Ireland. Therefore, spore identification, removal, and inactivation in drinking water and wastewater treatment processes remain technological challenges (Graczyk \& Lucy 2007).

Microsporidia were included in the United States Environmental Protection Agency's Contaminant Candidate List in 1998 and 2005 (Guillot \& Loret 2009), and are currently considered as Category B priority pathogens by the National Institute of Allergy and Infectious Diseases (NIAID, National Institutes of Health, Bethesda, MD, USA - niaid.nih.gov/topics/biodefenserelated/ biodefense/pages/cata.aspx) due to their impact on public health. However, the Brazilian Drinking Water Legislation (MS 2011) (Federal Decree 2914/Health Ministry) published in 2011 did not address microsporidia monitoring in the water supply.

The presence of $E$. bieneusi spores in the final effluent reported in this study suggests that $E$. bieneusi contamination by human sources may be occurring at the Atibaia River basin. Thus, enteropathogen sourcetracking research in wastewater treatment plants is critical, considering that surface water supplies in Brazil receive large amounts of raw sewage (Sodré et al. 2010).

\section{ACKNOWLEDGEMENTS}

To Mônica Santín-Duran, of the United States Department of Agriculture (Washington, DC, USA), for support in confirmation of the novel Enterocytozoon bieneusi genotype.

\section{AUTHORS' CONTRIBUTION}

SY - Conceived the study, participated in its design, conducted the study, conducted the analysis of laboratory tests, and participated in data acquisition and drafting of the manuscript; VRSF - conducted the phylogenetic analysis, and participated in data acquisition and drafting of the manuscript; ATLST, NB, CEL and ICVSC - participated in data acquisition; RMBF - conceived the study, participated in its design, conducted the study, and participated in data acquisition and drafting of the manuscript.

\section{REFERENCES}

Aikawa NE, Twardowsky AO, Carvallho JF, Silva CA, Silva IL, Ribeiro AC, et al. Intestinal microsporidiosis: a hidden risk in rheumatic disease patients undergoing anti-tumor necrosis fator therapy combined with disease-modifying anti-rheumatic drugs? Clinics. 2011; 66(7): 1171-5.

Ayed LB, Yang W, Widmer G, Cama V, Ortega Y, Xiao L. Survey and genetic characterization of wastewater in Tunisia for Cryptosporidium spp., Giardia duodenalis, Enterocytozoon bieneusi, Cyclospora cayetanensis and Eimeria spp. J Water Health. 2012; 10(3): 431-44.

Brasil P, de Lima DB, de Paiva DD, Lobo MS, Sodré FC, Silva SP, et al. Clinical and diagnostic aspects of intestinal microsporidiosis in HIV-infected patients with chronic diarrhea in Rio de Janeiro, Brazil. Rev Inst Med Trop São Paulo. 2000; 42(6): 299-304.

Brasil P, de Paiva DD, de Lima DB, da Silva EJ, Peralta JM, da Silva AJ, et al. A 3-year follow-up of a Brazilian AIDS patient with protracted diarrhea caused by Enterocytozoon bieneusi. Rev Inst Med Trop São Paulo. 1998; 40(4): 215-8.

Brasil P, Sodré FC, Cuzzi-Maya T, Gutierrez Mc, Mattos H, Moura H. Intestinal microsporidiosis in HIV-positive patients with chronic unexplained diarrhea in RJ, Brasil: diagnosis, clinical presentation and follow-up. Rev Inst Med Trop São Paulo. 1996; 38(2): 97-102.

Buckholt MA, Lee JH, Tzipori S. Prevalence of Enterocytozoon bieneusi in swine: an 18-month survey at a slaughterhouse in Massachusetts. Appl Environ Microbiol. 2002; 68(5): 2595-9.

Cantusio Neto R, Santos LU, Franco RMB. Evaluation of activated sludge treatment and the efficiency of the disinfection of Giardia species cysts and Cryptosporidium oocysts by UV at a sludge treatment plant in Campinas, Southeast Brazil. Water Sci Technol. 2006; 54(3): 89-94.

Cheng H-W A, Lucy FE, Graczyk TK, Broaders MA, Mastitsky SE. Municipal wastewater treatment plants as removal systems and environmental sources of human-virulent microsporidian spores. Parasitol Res. 2011; 109(3): 595-603.

Costa LNG, Marson FAL, Teixeira ATLS, Sales CRM, Levy CE. Intestinal microsporidiosis in a reference center. Letter to the editor. Braz J Infect Dis. 2013; 17(6): 724-5.

Cotte L, Rabodonirina M, Chapuis F, Bailly F, Bissuel F, Raynal C, et al. Waterborne outbreak of intestinal microsporidiosis in persons with and without human immunodeficiency virus infection. J Infect Dis. 1999; 180(6): 2003-8.

Cunha MJR, Cury MC, Santín M. Widespread presence of humanpathogenic Enterocytozoon bieneusi genotypes in chickens. Vet Parasitol. 2016; 217: 108-12. 
de Souza Jr ES, García-Zapata MT. Laboratory diagnosis of opportunistic intestinal parasites with emphasis on human microsporidiosis, in Goiânia/GO. Rev Soc Bras Med Trop. 2006; 39(6): 560-4.

Decraene V, Lebbad M, Botero-Kleiven S, Gustavsson A-M, Löfdahl M. First reported foodborne outbreak associated with microsporidia, Sweden, October 2009. Epidemiol Infect. 2012; 140(3): 519-27.

Didier ES, Storvall ME, Green LC, Brindley PJ, Sestak K, Didier PJ. Epidemiology of microsporidiosis: sources and modes of transmission. Vet Parasitol. 2004; 126(1-2): 145-66.

Didier ES, Weiss LM. Microsporidiosis: current status. Curr Opin Infect Dis. 2006; 19(5): 485-92.

Didier ES, Weiss LM. Microsporidiosis: not just in AIDS patients. Curr Opin Infect Dis. 2011; 24(5): 490-5.

Dowd SE, Gerba CP, Pepper IL. Confirmation of the human-pathogenic microsporidia Enterocytozoon bieneusi, Encephalitozoon intestinalis, and Vittaforma corneae in water. Appl Environ Microbiol. 1998; 64(9): 3332-5.

Fayer R, Santín-Duran M. Epidemiology of microsporidia in human infections. In: Weiss LM, Becnel JJ, editors. Microsporidia: pathogens of opportunity. Chichester: John Wiley \& Sons, Inc.; 2014. p. 135-64.

Feng Y, Li N, Dearen T, Lobo ML, Matos O, Cama V, et al. Development of a multilocus sequence typing tool for high-resolution genotyping of Enterocytozoon bieneusi. Appl Environ Microbiol. 2011; 77(14): 4822-8.

Field AS, Milner Jr DA. Intestinal microsporidiosis. Clin Lab Med. 2015; 35(2): 445-59.

Fiuza VRS, Lopes CWG, Cosendey RIJ, Oliveira FCR, Fayer R, Santín M. Zoonotic Enterocytozoon bieneusi genotypes found in Brazilian sheep. Res Vet Sci. 2016b; 107: 196-201.

Fiuza VRS, Lopes CWG, Oliveira FCR, Fayer R, Santín M. New findings of Enterocytozoon bieneusi in beef and dairy cattle in Brazil. Vet Parasitol. 2016a; 216: 46-51.

Fiuza VRS, Oliveira FCR, Fayer R, Santín M. First report of Enterocytozoon bieneusi in pigs in Brazil. Parasitol Int. 2015; 64(4): 18-23.

Fournier S, Dubrou S, Liguory O, Gaussin F, Santillana-Hayat M, Sarfati C, et al. Detection of microsporidia, cryptosporidia and Giardia in swimming pools: a one-year prospective study. FEMS Immunol Med Microbiol. 2002; 33(3): 209-13.

Franco RMB, Rocha-Eberhardt R, Cantusio Neto R. Occurrence of Cryptosporidium oocysts and Giardia cysts in raw water from the Atibaia River, Campinas, Brazil. Rev Inst Med Trop São Paulo. 2001; 43(2): 109-19.

Galván AL, Magnet A, Izquierdo F, Fenoy S, Rueda C, Vadillo CF, et al. Molecular characterization of human-pathogenic microsporidia and Cyclospora cayetanensis isolated from various water sources in Spain: a year-long longitudinal study. Appl Environ Microbiol. 2013; 79(2): 449-59.

Galván AL, Sánchez AMM, Pérez-Valentin MA, Henriques-Gil N, Isquierdo F, Fenoy S, et al. First cases of microsporidiosis in transplant recipients in Spain and review of the literature. J Clin Microbiol. 2011; 49(4): 1301-6.

Gerba CP, Riley KR, Nwachuku N, Ryu H, Abbaszadegan M. Removal of Encephalitozoon intestinalis, calicivirus, and coliphages by conventional drinking water treatment. J Environ Sci Health A Tox Hazard Subst Environ Eng. 2003; 38(7): 1259-68.

Graczyk TK, Lucy FE, Tamang L, Miraflor A. Human enteropathogens load in activated sewage sludge and corresponding sewage sludgeend products. Appl Environ Microbiol. 2007; 73(6): 2013-15.
Graczyk TK, Lucy FE. Quality of reclaimed waters: a public health need for source tracking of wastewater-derived protozoan enteropathogens in engineered wetlands. Trans R Soc Trop Med Hyg. 2007; 101(6): 532-3.

Graczyk TK, Sunderland D, Awantang GN, Mashinski Y, Lucy FE, Graczyk Z, et al. Relationships among bather density, levels of human waterborne pathogens, and fecal coliform counts in marine recreational beach water. Parasitol Res. 2010; 106(5): 1103-8.

Guillot E, Loret J-F. Waterborne pathogens: review for the drinkingwater industry. IWA Publishing. 2009; 194 pp.

Guo Y, Alderisio KA, Yang W, Cama V, Feng Y, Xiao L. Specificity and source of Enterocytozoon bieneusi genotypes in a drinking source watershed. Appl Environ Microbiol. 2014; 80(1): 218-25.

Henriques-Gil N, Haro M, Izquierdo F, Fenoy S, del Águila C. Phylogenetic approach to the variability of the microsporidian, Enterocytozoon bieneusi and its implications for inter- and intrahost transmission. Appl Environ Microbiol. 2010; 76(10): 3333-42.

Hu Y, Feng Y, Huang C, Xiao L. Occurrence, source, and human infection potential of Cryptosporidium and Enterocytozoon bieneusi in drinking source water in Shanghai, China, during a pig carcass disposal incident. Environ Sci Technol. 2014; 48(24): 14219-27.

Kumar S, Stecher G, Tamura K. Mega 7: molecular evolutionary genetics analysis version 7.0 for bigger datasets. Mol Biol Evol. 2016; 33(7): 1870-4.

Lallo MA, Calábria P, Bondan EF, Milanelo L. Identification of Encephalitozoon and Enterocytozoon (microsporidia) spores in stool and urine samples obtained from free-living South American Coatis (Nasua nasua). Appl Environ Microbiol. 2012a; 78(12): 4490-2.

Lallo MA, Calábria P, Milanelo L. Encephalitozoon and Enterocytozoon (microsporidia) spores in stool from pigeons and exotic birds. Microsporidia spores in birds. Vet Parasitol. 2012b; 190(3-4): 418-22.

Li J, Qi M, Chang Y, Wang R, Li T, Dong H, et al. Molecular characterization of Cryptosporidium spp., Giardia duodenalis, and Enterocytozoon bieneusi in captive wildlife at Zhengzhou Zoo, China. J Eukaryot Microbiol. 2015; 62(6): 833-9.

Li N, Xiao L, Wang L, Zhao S, Zhao X, Duan L, et al. Molecular surveillance of Cryptosporidium spp., Giardia duodenalis, and Enterocytozoon bieneusi by genotyping and subtyping parasites in wastewater. PLoS Negl Trop Dis. 2012; 6(9): e1809.

Li X, Palmer R, Trout JM, Fayer R. Infectivity of microsporidia spores stored in water at environmental temperatures. J Parasitol. 2003; 89(1): 185-8.

Ma J, Feng Y, Hu Y, Villegas EN, Xiao L. Human infective potential of Cryptosporidium spp., Giardia duodenalis and Enterocytozoon bieneusi in urban wastewater treatment plant effluents. J Water Health. 2016; 14(3): 411-23.

MS - Ministério da Saúde. Portaria 2914/MS. 2011. Available from: http://bvsms.saude.gov.br/bvs/saudelegis/gm/2011/ prt2914_12_12_2011.html.

Saitou N, Nei M. The neighbor-joining method: a new method for reconstructing phylogenetic trees. Mol Biol Evol. 1987; 4(4): 406-25.

Santín M, Fayer R. Microsporidiosis: Enterocytozoon bieneusi in domesticated and wild animals. Res Vet Sci. 2011; 90(3): 363-71.

Santín M, Vecino JAC, Fayer R. A zoonotic genotype of Enterocytozoon bieneusi in horses. J Parasitol. 2010; 96(1): 157-61.

Sodré FF, Locatelli MAF, Jardim WT. Occurrence of emerging contaminants in Brazilian drinking waters: a sewage-to-tap issue. Water Air Soil Poll. 2010; 206(1): 57-67.

Sparfel JM, Sarfati C, Liguory O, Caroff B, Dumoutier N, Gueglio B, 
et al. Detection of microsporidia and identification of Enterocytozoon bieneusi in surface water by filtration followed by specific PCR. J Eukaryot Microbiol. 1997; 44(6): 78S.

ten Hove RJ, van Leshout L, Beadsworth MBJ, Perez MA, Spee K, Claas ECJ, et al. Characterization of genotypes of Enterocytozoon bieneusi in immunosuppressed and immunocompetent patient groups. J Eukaryot Microbiol. 2009; 56(4): 388-93.

Vávra J, Lukes J. Microsporidia and "the art of living together". Adv Parasitol. 2013; 82: 253-319.

Weiss LM, Becnel JJ. Microsporidia: pathogens of opportunity. 1st ed. United Kingdom: John Wiley \& Sons, Inc; 2014. 709 pp.
Weiss LM. Clinical syndromes associated with microsporidiosis. In: Weiss LM, Becnel JJ, editors. Microsporidia: pathogens of opportunity. 1st ed. United Kingdom: John Wiley \& Sons, Inc; 2014. p. $371-401$.

Wuhib T, Silva TM, Newman RD, Garcia LS, Pereira ML, Chaves $\mathrm{CS}$, et al. Cryptosporidial and microsporidial infections in human immunodeficiency virus-infected patients in northeastern Brazil. J Infect Dis. 1994; 170(2): 494-7.

Wumba R, Longo-Mbenza B, Menotti J, Mandina M, Kintoki F, Situakibanza NH, et al. Epidemiology, clinical, immune, and molecular profiles of microsporidiosis and cryptosporidiosis among HIV/AIDS patients. Int J Gen Med. 2012; 5: 603-11. 